\title{
Monocyte Count in Preterm Neonates With and Without Necrotizing Enterocolitis
}

\author{
Saleheh Tajalli, PhD'; Fatemeh Erteghaee, MD²; Nasrin Hosseiny Nejad, MD²; Nasrin Khalesi, MD²; Leila Allahqoli, MPH \\ ${ }^{1}$ Nursing Care Research Center (NCRC), School of Nursing and Midwifery, Iran University of Medical Sciences, Tehran, Iran \\ ${ }^{2}$ Department of Neonatology, Ali Asghar Hospital, Iran University of Medical Sciences, Tehran, Iran \\ Iran University of Medical Sciences (IUMS), Tehran, Iran
}

\begin{abstract}
Background: Recently, blood monocyte concentration has been introduced as an inventive biomarker for necrotizing enterocolitis (NEC) in low birth weight neonate; therefore, we aimed to examine and compare blood monocyte counts in preterm neonates with and without NEC.

Methods: A case-control study was conducted on 160 preterm neonates in Tehran during 2017. In this study, medical records and laboratory findings of 80 neonates with NEC (case) and 80 neonates (control), who were randomly chosen from among neonates who had survived NEC, were reviewed and CBC outcomes were compared between the groups.

Results: We compared 80 cases and 80 controls. The mean gestational age in the NEC and control group was $30.78 \pm 2.33$ and $31.41 \pm 2.16$ weeks, respectively. The birth weights in the NEC and control group were $1500 \pm 488$ and $1562 \pm 360$ g, respectively. The mean age for developing NEC was $14.14 \pm 13.9$ days. The absolute neutrophil count (ANC) and absolute lymphocyte count (ALC) did not differ statistically significantly between the two groups. The mean absolute white blood cells (WBC) in the NEC and control group were $11368 \pm 7957$ and $8268 \pm 7505$, respectively; absolute WBC was significantly different between the two groups $(P=0.001)$. The mean absolute monocyte count $(A M C)$ in the NEC and control group were $262 \pm 193$ and $518 \pm 691$, respectively; the difference in absolute WBC was significant between the two groups $(P=0.002)$. Receiver operating characteristic of AMC values showed a diagnostic accuracy of $0.693(95 \% \mathrm{Cl}$ : $0.612-0.773)$ for NEC and $0.738(95 \% \mathrm{Cl}: 0.627-0.850)$ for stage II and III NEC.

Conclusion: The result of this study suggest that the concentration of blood monocyte could be an indicator for NEC in the preterm neonate. However, this result should be confirmed by other studies.

Keywords: Monocyte count, Necrotizing enterocolitis, Preterm neonate

Cite this article as: Tajalli S, Erteghaee F, Hosseiny Nejad N, Khalesi N, Allahqoli L. Monocyte count in preterm neonates with and without necrotizing enterocolitis. Arch Iran Med. 2022;25(1):26-31. doi: 10.34172/aim.2022.05
\end{abstract}

Received: November 6, 2020, Accepted: February 7, 2021, ePublished: January 1, 2022

\section{Introduction}

Necrotizing enterocolitis (NEC) is one of the most serious and common gastrointestinal medical/surgical emergencies in preterm neonates, which affects $6 \%-7 \%$ of extremely low birth weight neonates. NEC is one of the major causes of mortality for neonates born prior to 32 weeks of gestation or with birth weight less than 1500 grams. ${ }^{1-3}$ NEC diagnosis is based on radiological findings such as intestinal pneumatosis and portal venous gas, specific abdominal symptoms and clinical course, as well as pathologic findings, such as inflammation and coagulative (ischemic) necrosis and bacterial overgrowth. Unfortunately, the sensitivity of the mentioned methods is low and delayed in NEC. ${ }^{4}$ In pathology, NEC is characterized by infiltration of leukocytes and macrophages, which differs from other causes of intestinal dysfunction in infants such as motor disorder, disseminated sepsis-induced ileus and ischemia. ${ }^{5}$ The blood concentration of eosinophils and monocytes is part of a complete blood count. The reference range for this concentration has been published during the neonatal period, with high sample sizes and new methods that are needed to detect abnormal cases. ${ }^{6}$

The preterm neonate refers to a neonate with a gestational age of fewer than 37 weeks. Although the preterm neonate birth rate appears to vary by geographical area, reports show an incidence of $6 \%-10 \%$. Premature birth and related problems are the leading cause of morbidity and mortality during the first year of life. ${ }^{7}$ The preterm neonate has a limited count of circulating monocytes, and no significant reserves of adult monocytes are present in the bone marrow. ${ }^{6}$ Accordingly, a study was conducted with the hypothesis of increasing intestinal monocyte establishment during NEC and decreasing monocyte blood concentrations in VLBW (very low birth weight) infants with NEC and other nutritional intolerance problems, with the aim that this monocytic decline could be a diagnostic biomarker for NEC. The medical records of VLBW infants under treatment due to NEC were evaluated and comparison was done with non-NEC neonates with nutritional intolerance as the control group. Clinical criteria and purity of monocytes were recorded. In total, 69 cases and 257 controls were examined and compared within 26 to 29 weeks, and finally, blood 
monocyte depletion was introduced as a new biomarker for the diagnosis of NEC in VLBW neonates. ${ }^{8}$

Awareness of diagnostic problems and lack of a valid and measurable biomarker for early diagnosis of NEC forces specialists to treat neonates with abdominal and perilous symptoms, which is an expensive and unpleasant approach. ${ }^{9}$ There is a high prevalence of NEC in preterm neonates and this disease has been recognized as one of the essential reasons for their morbidity and mortality. ${ }^{10}$ There is an absence of accurate and effective methods for diagnosis, necessitating appropriate and rapid diagnostic methods to prevent unnecessary treatments. ${ }^{9}$

Early intervention and interventional therapy can improve clinical outcomes, but worrying disabilities still occur following NEC. ${ }^{2}$ Consequently, a study design (capable of measuring differences in the monocyte counts in preterm neonates with and without NEC) can be an effective step towards proving or rejecting the hypothesis of reduced blood monocyte count due to the increased incidence of intestinal monocyte establishment following regional and genetic conditions in the Iranian neonates. Such a study can also be effective in appropriately treating and reducing the mortality rate of these neonates. Therefore, this paper aims at examining and comparing preterm blood monocyte counts in preterm neonates with and without NEC in Ali Asghar hospital during 2016-2017.

\section{Materials and Methods}

This retrospective case-control study was conducted at Ali Asghar hospital affiliated to the Iran University of Medical Sciences (IUMS), Tehran, Iran in 2017. The study was approved by the Ethics Committee at (IR. IUMS. REC.1395.8811215234). A sample size of 80 neonates was calculated for each group ( $\alpha=0.05$, power: 0.09$)$ based on the results of similar studies. ${ }^{8}$ All neonates with a gestational age of less than 37 weeks who were admitted in the neonatal intensive care unit (NICU) between March 2010 and September 2017, were potentially eligible for the study. Neonates with congenital or acquired anomalies of the gastrointestinal tract (omphalocele, gastroschisis, tracheoesophageal, gastrointestinal perforation, intestinal obstruction), and incomplete medical records were excluded from the study.

The cases were 80 neonates with a NEC diagnosis (Bell stages II or III $)^{11}$ and, for each case, one control was selected based on birth weight $( \pm 200 \mathrm{~g})$, date of admission ( \pm 3 months), gestational age ( \pm 1 week), and age of NEC diagnosis. (One neonate with the above characteristics was selected for the control group at the age of diagnosis of NEC.) The control group comprised patients who were randomly chosen from among hospitalized neonates at the NICU of Ali Asghar hospital for medical reasons other than NEC before hospital discharge. The characteristics of the $\mathrm{CBC}$ outcomes were compared between case and control groups.

A checklist was prepared from the medical records of the neonates, including demographic profiles: gender, gestational age, delivery mode (normal vaginal delivery [NVD] or cesarean section), birth weight, and race; clinical characteristics: Apgar score, signs of disseminated sepsis, patent ductus arteriosus (PDA), intracranial hemorrhage (ICH) and starting age of NEC; and information from the complete blood count $(\mathrm{CBC})$ : the time of the test, the count of white blood cells (WBC), the absolute neutrophil count (ANC), the absolute lymphocyte count (ALC) and the absolute monocyte count (AMC) as the NEC-dependent variables. The obtained data was recorded completely in the checklist, which included the mean blood cell count in two blood tests before the onset of NEC symptoms and in neonates with only one test prior to diagnosis, and information from a test with the mean blood cell count in two blood tests in neonates without NEC. The CBC, as a commonly performed blood test, is often used for routine check-up and we had no induced test. The Siemens-Bayer ADVIA 2120 automated hematology counters were used to perform the clinical laboratory tests.

Ali Asghar hospital is an educational medical hospital with level IV referral NICU, which admits infants requiring all medical, surgical and neurosurgical interventions except cardiac surgery from all over the country. Also, there are facilities for sophisticated procedures, imaging evaluation or consultation with other pediatrics subspecialties, metabolic disorders, congenital malformations, hypoxic-ischemic encephalopathy (HIE), and neonatal seizure.

\section{Statistical Analysis}

Data were entered to the SPSS software version 22 and analysis was done. Mean $\pm S D$ were reported for quantitative variables. Qualitative variables were described by frequency (percentage). Data were also tested for distributed normality using Shapiro-Wilk test, and were found to be normally distributed. Qualitative variables were compared by student $t$ test and chi-square. The receiver-operator characteristics (ROC) of AMC values were evaluated by the ROC curve to assess sensitivity, false-positive rate, and utility of a diagnostic test. The usefulness of the test was assessed by the area under the ROC curve (AUC). The best statistical cut-off value for AMC was estimated based on ROC analysis. The point on the ROC curve where the sensitivity and specificity of the test were equal was considered as the best cut-off. This point of the curve is the line connecting the left-upper and the right lower corner of the unit square and the ROC curve and the product of this sensitivity and specificity is maximum at this point. The significance level was set at less than 0.05 .

\section{Results}

We reviewed all medical records from all 1952 neonates with a gestational age of less than 37 weeks who were admitted in NICU between March 2010 and September 2017 and identified 86 neonates with a diagnosis of NEC. Six neonates were excluded because of incomplete medical 
records. Thus, in final analysis, 80 neonates with confirmed NEC were included. Out of the 160 neonates examined, 89 (55.6\%) were boys and 71 (44.4\%) were girls; 155 (96.9\%) were Iranians and $5(3.1 \%)$ were Afghans. The minimum gestational age for neonates was 26 weeks, and the highest gestational age was 36 weeks. The mean gestational age for the neonates was $31 \pm 2.4$ weeks. The weight of the neonates was between $600 \mathrm{~g}$ and $3000 \mathrm{~g}$, with an average of $1531 \pm 428 \mathrm{~g}$. Out of 160 preterm neonates, 38 (23.75\%) and $122(76.25 \%)$ were born to NVD and cesarean section, respectively. The minimum age for developing NEC was one day and the highest age was 84 days with an average of $14.14 \pm 13.9$ days. The clinical and demographic characteristics of the neonates are summarized in Table 1.

According to the results of the study, the relationship between NEC and gender, gestational age, delivery mode, and birth weight was not statistically significant. Out of 80 neonates with NEC, 13 (16.25\%) had PDA, 4 (5\%) had ICH and 35 (43.75\%) had sepsis. The relationship between PDA and ICH with NEC was not significant, but there was a statistically significant relationship between sepsis and NEC $(P=0.001)$ (Table 1$)$.

According to the findings, WBC and ALC were non significantly higher in neonates with Bell stage III than stage II. However, ANC and monocyte counts in neonates with Bell stage III were significantly lower than stage II $(P=0.002)(P=0.001)$. As shown in Table 2 , ANC and ALC did not differ statistically significantly in the two groups, but the difference in $\mathrm{WBC}$ and $\mathrm{AMC}$ was significant between the groups with and without NEC $(P=0.001$, $P=0.002$ ) (Table 2).

In distinguishing NEC, ROC analysis was used to evaluate marker diagnostic performance. Table 3 shows the areas under the ROC (ROC-AUCs) curves for AMC values for NEC diagnosis and Bell stages II and III. ROC of AMC values showed a diagnostic accuracy (area under the curve) of 0.693 (95\% confidence interval [CI]: 0.6120.773 ) for NEC and 0.738 (95\% CI; 0.627-0.850) for stage II and III NEC (Figures 1 and 2).

\section{Discussion}

NEC is the one of most serious and common gastrointestinal emergency in preterm neonates and the absence of a reliable and measurable biomarker for early diagnosis of NEC forces specialists to treat neonates with abdominal and perilous symptoms, which is an expensive and unpleasant approach. ${ }^{9}$ Therefore, this cross-sectional analytical study was designed to compare blood monocyte counts in preterm neonates with and without NEC in Ali Asghar hospital during 2016-2017.

Based on the results of this study, the monocyte level was lower in the NEC group compared to the control group. To date, intestinal fatty acid-binding protein, the inter-alpha inhibitor protein, hexosaminidase, proapolipoprotein CII, and serum amyloid A have been identified as biomarkers for detection of NEC. ${ }^{12-17}$ The

Table 1. Characteristics of Neonates

\begin{tabular}{|c|c|c|c|c|}
\hline Characteristics & & $\begin{array}{l}\text { NEC Group } \\
\quad(\mathbf{n = 8 0})\end{array}$ & $\begin{array}{l}\text { Control Group } \\
\qquad(n=80)\end{array}$ & $P$ Value \\
\hline \multirow{2}{*}{ Gender, n (\%) } & Female & $37(46.2)$ & $34(42.5)$ & \multirow{2}{*}{$0.633^{*}$} \\
\hline & Male & $43(53.8)$ & $46(57.5)$ & \\
\hline \multirow{4}{*}{ Gestational age (wk) } & $<30$ & $55(68.7)$ & $52(65)$ & \multirow{3}{*}{$0.23^{*}$} \\
\hline & $30-34$ & $21(26.3)$ & $22(27.5)$ & \\
\hline & $>34$ & $4(5)$ & $6(7.5)$ & \\
\hline & Mean \pm SD & $30.78 \pm 2.33$ & $31.41 \pm 2.16$ & $0.07^{* *}$ \\
\hline \multirow{2}{*}{ Delivery mode, n (\%) } & NVD & $22(27.5)$ & $16(20)$ & \multirow{2}{*}{$0.265^{*}$} \\
\hline & $\mathrm{C} / \mathrm{S}$ & $58(72.5)$ & $64(80)$ & \\
\hline \multirow{4}{*}{ Birth weight } & $<1500$ & $58(72.5)$ & $50(62.5)$ & \multirow{3}{*}{$0.1^{*}$} \\
\hline & $1500-2500$ & $20(25)$ & $23(28.75)$ & \\
\hline & $>2500$ & $2(2.5)$ & $7(8.75)$ & \\
\hline & Mean \pm SD & $1500 \pm 488$ & $1562 \pm 360$ & $0.3^{* *}$ \\
\hline $\begin{array}{l}\text { Apgar score } 1 / 5 \\
\text { (min) }\end{array}$ & & $7.3 / 5.8$ & $7.6 / 8.8$ & $0.3^{* *}$ \\
\hline \multirow{3}{*}{ Comorbidities, n (\%) } & PDA & $13(16.25)$ & $14(17.5)$ & \multirow{3}{*}{$\begin{array}{l}0.889^{*} \\
0.687^{*} \\
0.001^{*}\end{array}$} \\
\hline & $\mathrm{ICH}$ & $4(5)$ & $3(3.75)$ & \\
\hline & Sepsis & $35(43.75)$ & $15(18.75)$ & \\
\hline $\begin{array}{l}\text { NEC Onset age (day) } \\
\text { Mean } \pm \text { SD }\end{array}$ & & 14.14(13.9) & - & - \\
\hline \multirow{2}{*}{ NEC stages } & II & $46(57.5)$ & - & - \\
\hline & III & $34(42.5)$ & - & - \\
\hline
\end{tabular}

SD, standard deviation; NVD, normal vaginal delivery; C/S, caesarean section; PDA, Patent ductus arteriosus; ICH, Intracranial hemorrhage; NEC, Necrotizing enterocolitis.

*Chi-squared test.

**Student's $t$ test. 
Table 2. CBC Outcomes on Day of NEC Diagnosis

\begin{tabular}{|c|c|c|c|c|c|c|c|c|}
\hline \multirow{2}{*}{\multicolumn{2}{|c|}{$\begin{array}{l}\text { Characteristics } \\
\text { NEC Stage }\end{array}$}} & & \multirow{2}{*}{$\begin{array}{c}\text { NEC } \\
(\mathbf{n}=\mathbf{8 0})\end{array}$} & \multirow{2}{*}{$\begin{array}{c}\text { Controls } \\
(\mathbf{n}=\mathbf{8 0})\end{array}$} & \multirow{2}{*}{$P$ value } & \multirow{2}{*}{$\begin{array}{c}\text { Mean } \\
\text { Difference }\end{array}$} & \multicolumn{2}{|c|}{ Confidence Interval $(95 \%$} \\
\hline & & & & & & & Lower & Upper \\
\hline \multirow{4}{*}{ WBC } & II & \multirow{3}{*}{ Mean \pm SD } & $10547 \pm 5090$ & \multirow{3}{*}{$8268 \pm 7505$} & \multirow{4}{*}{0.001} & \multirow{4}{*}{3100} & \multirow{4}{*}{1900} & \multirow{4}{*}{4300} \\
\hline & III & & $12479 \pm 10679$ & & & & & \\
\hline & Total & & $11368 \pm 7957$ & & & & & \\
\hline & & lax & $3300-54500$ & $2600-15800$ & & & & \\
\hline \multirow{4}{*}{ ANC } & II & \multirow{3}{*}{ Mean \pm SD } & $4503 \pm 2784$ & \multirow{3}{*}{$4463 \pm 3442$} & \multirow{4}{*}{0.1} & \multirow{4}{*}{699} & \multirow{4}{*}{500} & \multirow{4}{*}{760} \\
\hline & III & & $2764 \pm 1789$ & & & & & \\
\hline & Total & & $3764 \pm 2550$ & & & & & \\
\hline & & lax & $846-15210$ & $1011-27909$ & & & & \\
\hline \multirow{4}{*}{$\mathrm{ALC}$} & II & \multirow{3}{*}{ Mean \pm SD } & $3997 \pm 1781$ & \multirow{3}{*}{$4394 \pm 2499$} & \multirow{4}{*}{0.87} & \multirow{4}{*}{79} & \multirow{4}{*}{43} & \multirow{4}{*}{115} \\
\hline & III & & $5117 \pm 5478$ & & & & & \\
\hline & Total & & $4473 \pm 3828$ & & & & & \\
\hline & & lax & $770-32155$ & $240-16400$ & & & & \\
\hline \multirow{4}{*}{ AMC } & II & \multirow{3}{*}{ Mean \pm SD } & $319 \pm 205$ & & \multirow{4}{*}{0.002} & \multirow{4}{*}{256} & \multirow{4}{*}{186} & \multirow{4}{*}{326} \\
\hline & III & & $185 \pm 147$ & $518 \pm 691$ & & & & \\
\hline & Total & & $262 \pm 193$ & & & & & \\
\hline & & & $43-950$ & $88-5218$ & & & & \\
\hline
\end{tabular}

NEC, Necrotizing enterocolitis; WBC, white blood cells; ANC, absolute neutrophil count; ALC, absolute lymphocyte count; AMC, absolute monocyte count.

Table 3. ROC-AUCs Curves of AMC as a Test for NEC in Neonates

\begin{tabular}{lccc}
\hline NEC & $\boldsymbol{n}(\%)$ & ROC-AUC $(\mathbf{9 5} \% \mathbf{C I})$ & $\boldsymbol{P}$ Value \\
\hline NEC (Yes) & $80(50 \%)$ & 0.693 & $<0.001$ \\
NEC (NO) & $80(50 \%)$ & $(0.612-0.773)$ & \\
NEG stage II & $46(57.5)$ & 0.738 & $<0.001$ \\
NEG stage III & $34(42.5)$ & $(0.627-0.850)$ & \\
\hline
\end{tabular}

NEC, necrotizing enterocolitis; $\mathrm{N}$, number; $\mathrm{AMC}$, absolute monocyte count.

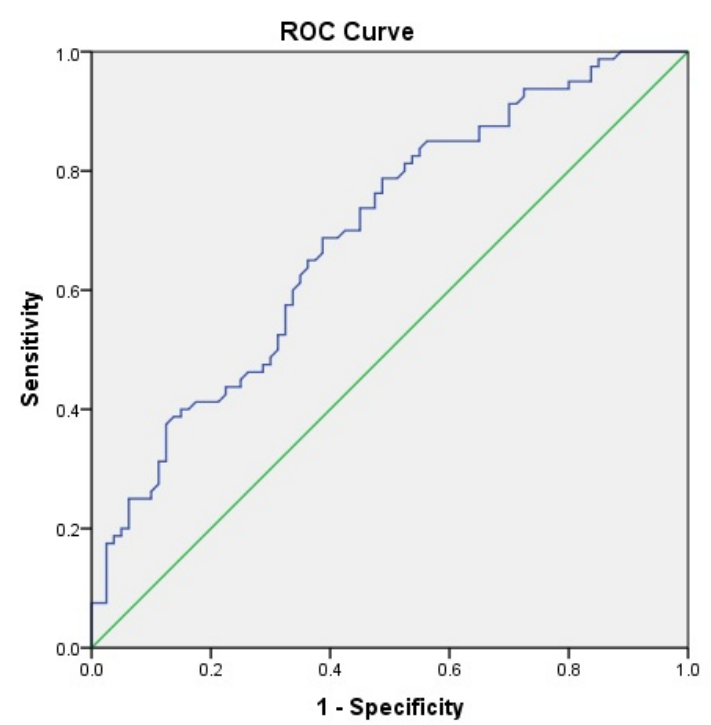

Diagonal segments are produced by ties.

Figure 1. Diagnostic Accuracy of Absolute Monocyte Count as a Test for Necrotizing Enterocolitis.

result of this research revealed that in preterm neonates, blood AMC may be a valuable diagnostic marker of NEC. The AMC is an appealing marker of NEC because

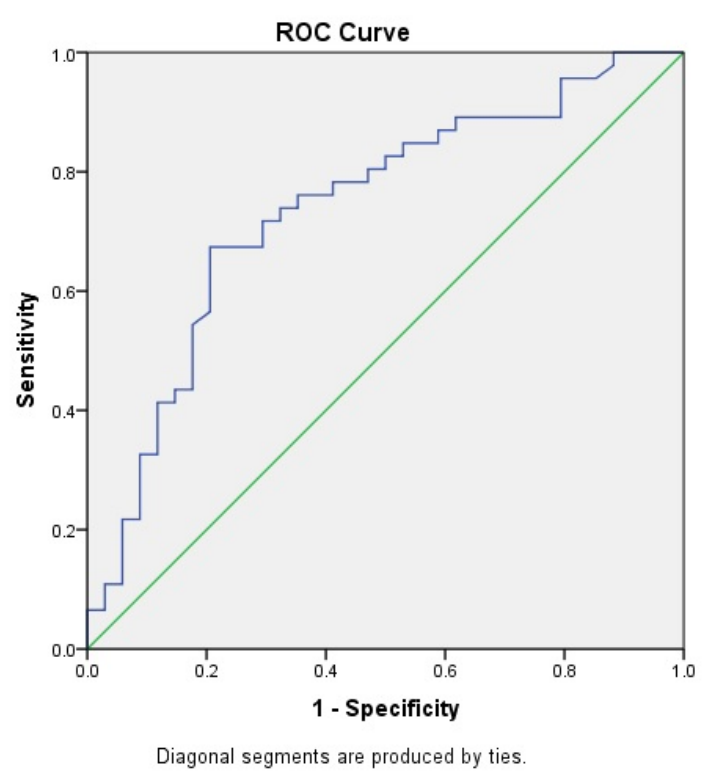

Figure 2. Diagnostic Accuracy of Absolute Monocyte Count as a Test for Stage of Necrotizing Enterocolitis.

the information is already available to the clinician at no additional cost. To our knowledge, few studies have shown that blood monocyte counts can serve as a diagnostic test for NEC. ${ }^{8,18,19}$

In the present study, monocyte counts were significantly lower in neonates with Bell stage III than stage II. Our preclinical NEC macrophage-rich infiltrate findings have contributed to our hypothesis that lower blood monocyte concentrations are correlated with NEC. ${ }^{20}$ Macrophages are produced from blood monocytes in inflammatory gastrointestinal lesions. ${ }^{20}$ In premature neonates, the rapid effluvium of monocytes for NEC lesions is likely to 
discharge the inadequate circulating pool of monocytes. ${ }^{20}$ Interestingly, reduced blood monocyte count is likely to be a unique trait of NEC. ${ }^{8}$ Similar to our study, a study conducted with the hypothesis of increased intestinal monocyte establishment during NEC and decreased blood monocyte counts among the VLBW neonates with NEC and other nutritional intolerance problems reported blood monocyte depletion as a new biomarker for detection of NEC in VLBW neonates. ${ }^{8}$

Monocytosis may occur more often than monocytopenia and can occur in combination with extreme prematurity, repeated transfusions of red blood cells, albumin, and theophylline. ${ }^{21}$ In syphilis and congenital candidiasis, ${ }^{22,23}$ as well as in immune-mediated neutropenia, monocytosis may also occur. ${ }^{24,25}$

This study has some limitations. (1) This study had a retrospective design which raises the possibility of bias. (2) This is a single-center study with a limited sample size $(n=180)$, which limits the generalizability of our results. It is recommended to conduct a future study with a large sample size and if possible, a study with a multicenter design to compare and confirm the obtained findings.

In conclusion, we found the ROC of AMC values with a diagnostic accuracy of 0.693 (95\% CI: 0.612-0.773) for NEC and 0.738 (95\% CI: 0.627-0.850) for stage II and III NEC. The result of this study suggests that a novel biomarker for NEC will be the concentration of blood monocytes in the preterm neonate. However, this result should be confirmed by other studies.

\section{Authors' Contribution}

ST, NHN, FE, NK, and LA conceptualized and designed the study, collaborated in data processing, collaborated in analysis. LA, ST, and NK wrote the manuscript, edited and critically reviewed manuscript. All authors read and approved the final manuscript.

\section{Conflict of Interest Disclosures}

There is no conflict to declare.

\section{Ethical Statement}

In this study, all ethical considerations were approved by ethics committee of Iran University of Medical Sciences (IUMS) (IR. IUMS. REC.1395.8811215234). Informed consent were obtained from all patients' parents.

\section{Acknowledgments}

We would like to thank Iran University of Medical Sciences, Tehran, Iran, for technical and financial support. Also, the authors wish to thank all the neonates' families who kindly agreed to participate in this study.

\section{References}

1. Rich BS, Dolgin SE. Necrotizing enterocolitis. Pediatr Rev. 2017;38(12):552-9. doi: 10.1542/pir.2017-0002.

2. Neu J. Necrotizing enterocolitis. World Rev Nutr Diet. 2014;110:253-63. doi: 10.1159/000358474.

3. Shulhan J, Dicken B, Hartling L, Larsen BM. Current knowledge of necrotizing enterocolitis in preterm infants and the impact of different types of enteral nutrition products. Adv Nutr. 2017;8(1):80-91. doi: 10.3945/an.116.013193.

4. Buonomo C. The radiology of necrotizing enterocolitis. Radiol Clin North Am. 1999;37(6):1187-98. doi: 10.1016/s0033-
8389(05)70256-6.

5. MohanKumar K, Kaza N, Jagadeeswaran R, Garzon SA, Bansal A, Kurundkar AR, et al. Gut mucosal injury in neonates is marked by macrophage infiltration in contrast to pleomorphic infiltrates in adult: evidence from an animal model. Am J Physiol Gastrointest Liver Physiol. 2012;303(1):G93-102. doi: 10.1152/ajpgi.00016.2012.

6. Christensen RD, Jensen J, Maheshwari A, Henry E. Reference ranges for blood concentrations of eosinophils and monocytes during the neonatal period defined from over 63000 records in a multihospital health-care system. J Perinatol. 2010;30(8):540-5. doi: 10.1038/jp.2009.196.

7. Balaraman V. Case Based Pediatrics for Medical Students and Residents. Chapter III.5. Common Problems of the Premature Infant. 2003. Available from: https://www.hawaii.edu/ medicine/pediatrics/pedtext/s03c05.html.

8. Remon J, Kampanatkosol R, Kaul RR, Muraskas JK, Christensen RD, Maheshwari A. Acute drop in blood monocyte count differentiates NEC from other causes of feeding intolerance. J Perinatol. 2014;34(7):549-54. doi: 10.1038/jp.2014.52.

9. D'Angelo G, Impellizzeri P, Marseglia L, Montalto AS, Russo T, Salamone I, et al. Current status of laboratory and imaging diagnosis of neonatal necrotizing enterocolitis. Ital J Pediatr. 2018;44(1):84. doi: 10.1186/s13052-018-0528-3.

10. Berman L, Moss RL. Necrotizing enterocolitis: an update. Semin Fetal Neonatal Med. 2011;16(3):145-50. doi: 10.1016/j. siny.2011.02.002.

11. Walsh MC, Kliegman RM. Necrotizing enterocolitis: treatment based on staging criteria. Pediatr Clin North Am. 1986;33(1):179-201. doi: 10.1016/s0031-3955(16)34975-6.

12. Ng PC, Ang IL, Chiu RW, Li K, Lam HS, Wong RP, et al. Hostresponse biomarkers for diagnosis of late-onset septicemia and necrotizing enterocolitis in preterm infants. J Clin Invest. 2010;120(8):2989-3000. doi: 10.1172/jci40196.

13. Chaaban H, Shin M, Sirya E, Lim YP, Caplan M, Padbury JF. Inter-alpha inhibitor protein level in neonates predicts necrotizing enterocolitis. J Pediatr. 2010;157(5):757-61. doi: 10.1016/j.jpeds.2010.04.075.

14. Edelson MB, Sonnino RE, Bagwell CE, Lieberman JM, Marks WH, Rozycki HJ. Plasma intestinal fatty acid binding protein in neonates with necrotizing enterocolitis: a pilot study. J Pediatr Surg. 1999;34(10):1453-7. doi: 10.1016/s00223468(99)90102-1.

15. Lobe TE, Richardson CJ, Rassin DK, Mills R, Schwartz M. Hexosaminidase: a biochemical marker for necrotizing enterocolitis in the preterm infant. Am J Surg. 1984;147(1):4952. doi: 10.1016/0002-9610(84)90033-3.

16. Lobe TE, Schwartz MZ, Richardson CJ, Rassin DK, Gourley WK, Srivastava SK, et al. Hexosaminidase: a marker for intestinal gangrene in necrotizing enterocolitis. J Pediatr Surg. 1983;18(4):449-52. doi: 10.1016/s0022-3468(83)80198-5.

17. Shattuck KE, Richardson CJ, Rassin DK, Lobe TE. Evaluation of hexosaminidase activity as a potential biochemical marker in serum for necrotizing enterocolitis. J Pediatr Gastroenterol Nutr. 1987;6(2):234-7. doi: 10.1097/00005176-19870300000013.

18. Maheshwari A. Immunologic and hematological abnormalities in necrotizing enterocolitis. Clin Perinatol. 2015;42(3):56785. doi: 10.1016/j.clp.2015.04.014.

19. Desiraju S, Bensadoun J, Bateman D, Kashyap S. The role of absolute monocyte counts in predicting severity of necrotizing enterocolitis. J Perinatol. 2020;40(6):922-7. doi: 10.1038/ s41372-020-0596-2.

20. Smythies LE, Maheshwari A, Clements R, Eckhoff D, Novak $\mathrm{L}, \mathrm{Vu} \mathrm{HL}$, et al. Mucosal IL-8 and TGF-beta recruit blood monocytes: evidence for cross-talk between the lamina propria stroma and myeloid cells. J Leukoc Biol. 2006;80(3):492-9. doi: 10.1189/jlb.1005566. 
21. Lee JS, Sperry IL, Ochoa JB, Barclay D, Namas R, Vodovotz Y, et al. Persistence of elevated plasma CXCL8 concentrations following red blood cell transfusion in a trauma cohort. Shock. 2012;37(4):373-7. doi: 10.1097/SHK.0b013e31824bcb72.

22. Shiou SR, Yu Y, Chen S, Ciancio MJ, Petrof EO, Sun J, et al. Erythropoietin protects intestinal epithelial barrier function and lowers the incidence of experimental neonatal necrotizing enterocolitis. J Biol Chem. 2011;286(14):12123-32. doi: 10.1074/jbc.M110.154625.

23. Raabe BM, Artwohl JE, Purcell JE, Lovaglio J, Fortman JD. Effects of weekly blood collection in C57BL/6 mice. J Am
Assoc Lab Anim Sci. 2011;50(5):680-5.

24. Rosebraugh MR, Widness JA, Nalbant D, Veng-Pedersen P. A mathematical modeling approach to quantify the role of phlebotomy losses and need for transfusions in neonatal anemia. Transfusion. 2013;53(6):1353-60. doi: 10.1111/j.1537-2995.2012.03908.x.

25. Guillén U, Cummings JJ, Bell EF, Hosono S, Frantz AR, Maier $\mathrm{RF}$, et al. International survey of transfusion practices for extremely premature infants. Semin Perinatol. 2012;36(4):2447. doi: 10.1053/j.semperi.2012.04.004. 